\title{
Topological field theories
}

\section{R. Rajaraman}

THEORETICAL high-energy physics throws up, every few years, newer and more complex formulations which we hapless soldiers in the field have to contend with and absorb. In the last five years, beginning with the reincarnation of String theory, life on this treadmill has become even more frantic, with an explosion of new theoretical structures and concepts. One such very recent concept is that of a Topological Field Theory (TFT).

This is not a topic that lends itself easily to a simple semi-technical level introduction. It is fairly abstract, dealing with ideas that at first sight appear to be more mathematical than physical, and was not designed a priori to model any specific experimental phenomenon. Nevertheless, it is a very interesting subject at the conceptual level and, like other elegant theoretical structures born ahead of their times, may eventually end up having important applications. Besides, TFT is a part of the larger subject of quantum field theories in curved space-time, which are already playing a major role in theoretical physics at both the microscopic and cosmological scales. We will therefore attempt to provide here some background on quantum field theories in curved spaces, sufficient for giving, at the end, a glimpse of what TFT is.

Quantum field theory deals with the quantum behavior of different types of waves, which, following the wave-particle duality inherent in quantum theory, are also expected to yield as their quanta some elementary particles. For several decades, such quantum field theories constructed to describe elementary particles were mostly couched in 'flat space-time'. Let us explain this remark. Consider that prototype example, the quantum theory of electromagnetic waves (free photons). These waves, called the 'fields' of this theory, are described by a set of four functions of space and time, $A_{\mu}(t, \vec{x})$, one for each value of the index $\mu=0,1,2$ or 3 . These $A_{\mu}$ are nothing but the familiar electromagnetic potentials we study in textbooks on classical electromagnetic theory. What is important to note for our purposes is that the underlying space-time continuum (labelled by the coordinates $(t, \vec{x})$ ) in which these fields propagate was taken to be flat-in the same sense that a table top is flat. This was done not merely for simplicity but also because the space-time on and near the earth, where laboratory experiments are done,

R. Rajaraman is in the Centre for Theoretical Studies, Indian Institute of Science, Bangalore 560012. is flat to a high degree of accuracy. For instance, to give one indicator of the flatness of space, if you set up in your room a triangle made of three meter-sticks, the sum of its angles will be very close to 180 degrees, unlike, say, 'triangles' made up of great-circle segments on the surface of a football. It is therefore reasonable that people used a flat space-time, not only for the theory of free photons, but also for the more complicated quantum field theories (collectively known as the Standard Model) which describe the electromagnetic, weak and strong interactions of all elementary particles. This is adequate for describing their terrestrial behavior.

Meanwhile, Einstein's General Theory of Relativity tells us that curvature of space-time is associated with the presence of gravitational forces. Using flat spacetime theories of elementary particles is equivalent to ignoring gravitational forces acting on them. Why is this permissible when direct personal experience tells us that there is very much a force of gravity due to the Earth permeating, among other places, every nook and cranny of every particle-physics laboratory? The reason is that the gravitational field due to the Earth is in fact intrinsically very small. In appropriate dimensionless units, the gravitational potential due to the earth at its surface is about $10^{-9}$. Furthermore it does not vary much over nuclear size scales. Hence individual elementary particles like protons feel only a tiny force of gravity due to the earth and even less due to one another. The other forces, nuclear and electromagnetic, that act on, say, a proton in a nucleus or in a collision experiment are overwhelmingly larger. (This assertion that the Earth's gravity is small may be indignantly contested by someone who has just fallen down the stairs or been struck on the head by a descending apple. But the admittedly large pull of gravity that you and I and the apple feel is because our own masses are large. By contrast for the tiny individual elementary particles in our experiments, the approximation of neglecting effects of gravity and of space-time curvature is quite justifiable.)

When is it that we really need field theories in curved space-time? Clearly, when the gravitational field is very large, and rapidly varying. Such strong gravity may be expected in the vicinity of very heavy and compact astronomical objects like black holes, or better still, in the universe in its early stages when all its mass is believed to have been concentrated in an extremely small volume, leading to awesome gravitational forces 
and huge distortions in spacetime. If one wished to study microscopic physics in such an environment then curved-space field theory becomes essential. Such studies gained popularity as people began to get interested in questions like particle production near black holes etc., and in quantitative attempts to model the early stages of our universe, its 'inflation' scenario and so on. Interest in the early universe was not limited to cosmologists alone. It was also shared by particlephysicists because of their widely held view that the somewhat complicated elementary particle interactions we find today could have originated from more symmetric and unified patterns in the early universe, thanks to some phase transitions. Field theories in curved manifolds also received a major impetus from the philosophical compulsion felt by particle-theorists that a complete quantum theory of fundamental interactions must include gravity in its purview. Further momentum came from superstring theory. A string, when it propagates, traces out a 2-dimensional curved surface, and its oscillations can be, viewed as wave fields on that surface. Motivated by the physics of all these important problems, a large body of work has emerged in the last two decades on quantum field theories in curved space-time and the subject has taken on a life of its own, with experts merrily pursuing its various ramifications. Studies were done not only on 4-dimensional space-time surfaces but also in 2,3 , as well as higher dimensions.

For illustration consider the Klein-Gordon system. In 4-dimensional flat space-time it obeys the familiar wave equation

$$
\left(\frac{\partial^{2}}{\partial t^{2}}-\vec{\nabla}^{2}\right) \phi(t, \vec{x})+m^{2} \phi(t, \vec{x})=0
$$

The Action which yields this equation, in relativistic notation, is

$$
S_{1}=\frac{1}{2} \int \mathrm{d}^{4} x\left(\eta^{\mu \nu} \partial_{\mu} \phi \partial_{\nu} \phi-m^{2} \phi^{2}\right)
$$

where $x^{\mu}(\mu=0,1,2,3)$ is the space-time coordinate,

$$
\begin{aligned}
\partial_{\mu} \phi & \equiv \frac{\partial \phi}{\partial x^{\mu}}, \\
\eta^{\mu \nu} & =0 \text { if } \mu \neq \nu, \\
\text { and } \eta^{00} & =-\eta^{11}=-\eta^{22}=-\eta^{33}=1 .
\end{aligned}
$$

The corresponding Action for the Klein-Gordon field in an arbitrary n-dimensional curved space-time is

$$
S_{2}=\frac{1}{2} \int \mathrm{d}^{n} x\left(\text { Det }\left|g_{\mu \nu}\right|\right)^{\frac{1}{2}}\left(g^{\mu \nu} \partial_{\mu} \phi \partial_{\nu} \phi-m^{2} \phi^{2}\right)
$$

where the tensor $g_{\mu v}(x)$ is the 'metric' of that curved space-time, which contains the geometrical information about that surface, such as its curvature at each point $x$ and so on. $g^{\mu \nu}$ is the matrix inverse of $g_{\mu v}$. The Action (3) has been designed to be invariant under any general transformation of the coordinates describing the surface, from $x^{\mu}$ to some $\left(x^{\prime}\right)^{\mu}$, provided all scalars, vectors, tensors etc. are also transformed by their appropriate transformation laws:

$$
\begin{aligned}
& \text { As } x^{\mu} \rightarrow\left(x^{\prime}\right)^{\mu}, \\
& \phi(x) \rightarrow \phi^{\prime}\left(x^{\prime}\right)=\phi(x) \\
& A_{\mu}(x) \rightarrow A_{\mu}^{\prime}\left(x^{\prime}\right)=\frac{\partial x^{\lambda}}{\partial x^{\prime \mu}} A_{\lambda}(x) \\
& g_{\mu v}(x) \rightarrow g_{\mu v}^{\prime}\left(x^{\prime}\right)=\frac{\partial x^{\lambda}}{\partial x^{\prime \mu}} \frac{\partial x^{\rho}}{\partial x^{\prime \nu}} g_{\lambda \rho}(x)
\end{aligned}
$$

This requirement of the invariance of the Action follows from the principle of Relativity. Recall that in Einstein's Special Theory of Relativity, physical laws are required to be the same if one goes from one coordinate frame to another which is moving at a uniform velocity with respect to the former frame. This change of frames corresponds to a special subset of coordinate transformations, namely, the Lorentz transformations. In General Relativity, all coordinate transformations are considered, including those which correspond to arbitrarily accelerated frames. It is straightforward algebra to verify that under such general coordinate transformations (4), the Action (3) is indeed unchanged.

As we all know, the sound waves emitted by a tabla depend, among other things, on the size and shape of its membrane. Similarly one would normally expect that the dynamics of any field theory should depend on the geometry of the underlying space-time. It is only reasonable that the physical content of a system, such as its correlation functions, the masses of its particles, their interactions etc. should change if the underlying space-time is bent, twisted, shrunk or deformed in some other way. For the Klein-Gordon example, such dependence on the geometry of its space-time is reflected by the presence of the metric $g_{\mu v}$, in both the Action (3) and the wave equation that emerges from it. This dependence of the dynamics on the metric $g_{\mu \nu}$ can also be interpreted in another way in accordance with General Relativity. Instead of interpreting the object $g_{\mu v}(x)$ in the Action (3) as the metric, it can be alternately viewed as an external gravitational field acting on the matter field $\phi$. The dynamics of the waves of $\phi$, or their quanta, will obviously depend on whatever external forces act on them, which in the present case stem from the gravitational field $g_{\mu v}$.

Usually, field theoretic Actions, if required as per General Relativity to be invariant under general coordinate transformations $x^{\mu} \rightarrow\left(x^{\prime}\right)^{\mu}$, will depend on the metric, as happened in the Klein-Gordon example. There are dozens of such examples involving scalar, spinor and vector fields. There are however, some exceptions, and that leads us at last to Topological Field Theories. 
First a capsule explanation of the word 'topological'. Two surfaces are said to be in the same topological class, or be topologically equivalent, if they can be converted from one to the other by smooth deformations. In this process of 'smooth deformations', any finite amount of stretching, pulling, twisting, bending and shrinking of the surface are all allowed, but not punching holes in it, nor cutting or tearing it, nor doing the opposite of tearing, i.e. pasting portions of it together. Using these guidelines one can see that the outer surfaces of a ball, an egg, and a cube are all topologically equivalent. Obviously two topologically equivalent surfaces must have the same dimensions, but this alone is not sufficient. For instance, the surface of a car tyre's tube (a torus) is not in the same class as the surface of a ball and neither is in the same class as a disc (a circle including its interior), though all these are 2-dimensional. Skeptical readers are invited to try and convert these into one another without violating the rules given above.

In this fashion all surfaces in any given number of dimensions can be divided into topologically distinct classes. Within the same class, two members have the same topology but they need not have the same geometry, i.e. the same shape, size, curvature etc. These geometrical features that distinguish two members within the same topological class, such as the surfaces of a sphere and an egg, are contained in the metric tensor $g_{\mu v}$, which will be different for the two surfaces. In turn, each topological class is characterised by topological invariants, which distinguish it from other classes. For instance, the surfaces of the ball and the egg have no holes or "handles" in them whereas the surface of a tyretube (and of a tea-cup which is topologically equivalent to it) carries one hole/handle.

Returning to field theories, we have seen that their dynamics usually does depend on their space-time geometry. There are however exceptional cases of systems, whose quantum dynamics does not depend on the geometry of the underlying space-time surface but only on the broad topological class to which it belongs. These are Topological Field Theories.

One way to try to get a TFT is to start with an Action which, although general coordinate invariant, does not depend on the metric $g_{\mu \nu}$. These are the TFT's of the 'Schwartz type', of which the prototype example is the abelian Chern-Simons Theory in 3 (two-spaceone-time) dimensions ${ }^{1-5}$. Its Action is

$$
S_{3}=\int \mathrm{d}^{3} x \varepsilon^{\lambda \mu \psi} A_{\lambda} \partial_{\mu} A_{\nu}
$$

Here $A_{\lambda}$ is a gauge field and $\varepsilon^{\lambda \mu \nu}$ is the fully antisymmetric Levi-Civita tensor. Even though the metric does not appear in (5), nor covariant derivatives of $A_{\lambda}$, that integral is in fact general relativistically invariant since it is unchanged under the transformations. $x^{\mu} \rightarrow\left(x^{\prime}\right)^{\mu}$, with $A_{\mu}(x) \rightarrow A_{\mu}^{\prime}\left(x^{\prime}\right)$ as per (4). The field equation arising from (5) is,

$$
\varepsilon^{\lambda \mu v} \partial_{\mu} A_{v} \equiv \frac{1}{2} \varepsilon^{\lambda \mu v} F_{\mu \nu}=0
$$

Since neither (5) nor (6) depend on the metric $g_{\mu \nu}$, if we changed the geometry of the underlying 3-dimensional space-time without changing its topology, the classical dynamics of this wave field $A_{\lambda}$ will not be affected at all. Of course we cannot extend this statement across different topologies. Surfaces with different topologies cannot even be covered by the same set and range of coordinates, so the question of fields having globally identical dynamics does not arise.

The metric independence of the Action (5) and the field equation (6) tells us that classically the system depends only on the topology of its space-time. This property could have conceivably broken down at the quantum level. To quantise this system, say, in the path integral formulation, one must add gauge fixing terms to the Action (5) which generally involve the metric. Also, the path integral measure could, in order to preserve general coordinate invariance, involve the metric. It has however been shown that for the 3 dimensional Chern-Simons theory, possible metric dependences from these sources cancel out ${ }^{6}$. In fancy language, this system has no 'topological anomaly'.

One can obtain other TFT's by generalising the abelian Chern-Simons system (5). For instance its nonabelian analogue is

$$
S_{4}=\frac{1}{4} \pi \int \mathrm{d}^{3} x \varepsilon^{\lambda \mu \nu} \operatorname{Tr}\left(A_{\lambda} \theta_{\mu} A_{\nu}+\frac{2}{3} A_{\lambda} A_{\mu} A_{\nu}\right)
$$

where $A_{\lambda}$ now is a matrix-valued Yang-Mills field. Generalisations can also be made to higher odddimensional Chern-Simons systems. For instance, abelian Chern-Simons theories in 5 dimensional spacetimes with and without boundaries have been studied in some detail ${ }^{7,8}$.

In the examples mentioned above, quantum TFT's have been obtained by starting from their classical counterparts, i.e. by the simple device of thinking up integrals (involving some fields) which, while being general coordinate invariant, nevertheless do not involve the metric. The so-called Witten-type of TFT's are even more remarkable in that their Actions do involve the metric of the underlying space-time, and yet the quantum theory is topological ${ }^{9}$. Their quantum partition functions do not depend on the metric. This happens whenever the system's Action is itself the variation under a 'BRST' transformation of some other Action. To those who are not familiar with what a 'BRST transformation' means, its only feature relevant for our purposes is that it is generated by a nilpotent fermionic charge. Yet another type of TFT, again studied in depth by Witten, is the Topological Sigma Model $^{10,11}$, where independence is demanded from the metric of not just the underlying space-time of the theory, but also that of the 'target space' i.e. the space 
of fields. We will be content with having just made a mention of these other types of TFT. To elaborate on them further, or to discuss the relationship of TFT's to other theoretical structures like Conformal field theories, Instantons etc., would require setting up more technical machinery than what would be appropriate for this article.

In topological field theories, all physical quantities will be topological invariants, i.e. objects constructed without using the metric. Take the simplest example, the abelian 3 dimensional Chern Simons theory (5). It is gauge invariant and its field equation (6) says that $F_{\mu m}$ the set of electric and magnetic fields written in relativistic notation, vanishes. Now, ordinarily (that is to say in familiar flat Minkowskian spacc-time) if the electric and magnetic fields vanished everywhere, there would be no physical degrees of freedom left from the $A_{\lambda}$. This, however, need not be true if the underlying 3 dimensional space-time has a complicated topology, with boundaries, holes etc. Then some degrees of freedom could survive, because there can exist some solutions of the differential equation (6) which cannot be galuge-transformed away. Such solutions will of course depend on what that topology is but not on the metric, which appears nowhere in (6).

Deep connections seem to exist between TFT's and aspects of modern mathematics. A prominent example involves the theory of 'knots', a preoccupation, once again, of Topologists. The basic theme of this theory is not hard to explain. Imagine some closed curve lying in 3 dimensions. It may not always be possible to spread this curve out into a nice circle without first snipping it - -it might have wound around itself a few times to form a knot. Such a knotted closed curve may also not be smoothly shrunk to a point-the knot would intervene. Not only is a knotted curve thus topologically different from an un-knotted one, but clearly there can be knots of different types and complexity, leading to questions of whether or not a given knotted curve can be deformed into another. A collection of several such curves weaving through one another form even more complicated entities called 'links'. Unravelling the mysteries of knots which cannot be unravelled is just the sort of thing mathematicians love. They have classified all such knots and links imbedded on 3 dimensions, in terms of certain 'knot-invariants'.

What does all this have to do with field theory? Well, in any quantum field theory one is primarily interested in correlation functions, i.e. vacuum expectation values of products of physical operators. Now take our 3 dimensional Chern-Simons theory (5). This is a TFT as well as a gauge theory. Physical quantities in this theory must be both gauge invariant and topologically invariant. The famous Wilson-loop operator

$$
W[C]=\exp .\left\{\oint_{C} A_{\mu} \mathrm{d} x^{\mu}\right\}
$$

provides such an object. It is gauge invariant and, being invariant under general coordinate transformations (4) without involving the metric, is also a topological invariant. This operator (and its non-abelian analogue) are widely used in gauge theories for many purposes including understanding 'quark confinement', and its correlation functions would in any case be of interest in any gauge theory. The line-integral in (8) is over some closed curve $\mathrm{C}$ lying in the 3-dimensional space-time of that Chern-Simons theory. Now we know from Stokes' theorem that if we deform this curve $C$ a little the line integral $\oint_{c} A_{\mu} \mathrm{d} x^{\mu}$ will be altered by the electromagnetic flux through the extra area-element swept by the curve during the deformation. However, since the electromagnetic field $F_{\mu v}$ vanishes for the Chern-Simons theory (see eq. (6)), this flux is zero. Hence smooth deformations will not alter the value of the Wilson loop $W(C)$. In particular, if the curve $C$ could be shrunk to a point, it means that $\oint_{c} A_{\mu} \mathrm{d} x^{\mu}$ vanishes and $W(C)=1$. It is only when the curve $C$ cannot be shrunk to a point, as for instance if it is knotted, that $W(C)$ will be nontrivial, with a value which can depend only on the topology of the curve $C$. Similarly the correlation function of a product of Wilson loops $\left\langle W\left(C_{1}\right) W\left(C_{2}\right)\right.$ $W\left(C_{3}\right) \ldots>$ can depend only on the topology of the knotting and mutual linking of these curves $C_{1}, C_{2}, C_{3}$ etc. These statements can be generalised to the nonabelian case (7). The relationship to the mathematics of knot theory is thus plausible. Witten showed ${ }^{1}$ that in fact the correlation functions of products of Wilsonloop operators in the Chern-Simons theory (7), when evaluated, yield precisely the knot invariants invented by mathematicians.

No physical applications have been suggested so far for TFT's as a class, applications which rely specifically on the property of their being topological. But the Chern-Simons system is already being used in a variety of contexts in physies. A prominent use is in the subject of Anyons-particles which are neither Bosons nor Fermions, but lie in between. Such beasts can exist in $2+1$ dimensions and can be produced from ordinary Bosons by coupling them to the $A_{\mu}$ of the ChernSimons system (5). And, Anyons in turn have been suggested as part of a mechanism to explain high- $T_{c}$ superconductivity ${ }^{12}$. Surely, as time goes on, topological field theories will have even more links with down to earth physics!

1. Witten. E.. Commun. Math. Phys., 1988, 117, 353.

2. Schwartz, A. S., Leti. Math. Phys., 1978, 2, 247.

3. Dunne, G. V., Jackiw, R. and Trugenberger, C. A., Ann. Phis. (N. Y). 1989, 194, 197.

4. Elitzur, S., Moore, G., Schwimmer, A. and Seiberg, N., Nucl. Phys., 1989, B326, 108.

5. Bos, M. and Nair, V. P., Phys. Lett., 1989, B223, 61. 
6. Kaul, R. K. and Rajaraman, R., to appear in Phys. Lett. B., 1990.

7. Floreanini, R. and Percacci, R., Phys. Lett., 1989, B224, 291.

8. Floreanini, R., Percacci, R. and Rajaraman, R., Phys. Lett., 1989, B231, 119.

9. Witten, E., Commun. Math. Phys., 1989, 121, 351.

10. Witten, E., Commun. Math. Phys, 1988, 118, 411.

11. Balieau, L. and Singer, I. M., The Topological Sigma Model, Paris preprint LPTHE 88/44, 1988.
12. Chen, Y., Wilczek, F., Witten, E. and Halperin, B., Int. J. Mod. Phys., 1989, B3, 1001 .

ACKNOWLEDGEMENT. I thank Dr. D. Sen for a critical reading of this manuscript. 